\title{
An Overview of a Blockchain Application in Education Using Hyperledger Project
}

\author{
Bouchaib Falah $^{1}$, Hamza Touhs ${ }^{1}$, Soufiane Karroumi ${ }^{1}$, Sameer Abufardeh ${ }^{2}$ \\ ${ }^{1}$ School of Science and Engineering, Al Akhawayn University, Morocco, ${ }^{2}$ Department of \\ Computer, Electrical, and Software Engineering, Embry-Riddle Aeronautical University, \\ USA.
}

\begin{abstract}
During the last years, blockchain has been getting interest by both academic and professional researchers. This is primarily due to the innovative techniques in which records are effectively stored in a distributed manner. Started as a value transfer mechanism, blockchain now has several applications in a vast array of fields including healthcare, banking, and Internet of Things (IoTs). Despite the considerable amount of interest given to these fields, little is known about the possible applications of blockchain in education. Because of COVID-19 outbreak and the rise in the need of online and automated solutions in academia, this paper proposes an overview of a blockchain solution in education. This technology relies on the unique features it offers, such as decentralization, data integrity, and security. The presented solution aims to reduce the paperwork needed to perform tasks, secure the stored data, increase transparency, as well as to offer new recruitment possibilities and statistics capabilities to the current educational system.
\end{abstract}

Keywords: Blockchain; education; transparency; reliability; security; academic career. 


\section{Introduction}

First emerging in 2008, by Dr. Satoshi Nakamoto (Nakamao, 2008), Blockchain's first implementation was a peer-to-peer shared ledger for cryptocurrency (Herbert et al, 2015). The focus of the technology was to decentralize systems and remove third-party intermediaries; thus, allowing direct peer-to-peer transactions (Barenji et al, 2018). This was possible thanks to the peer node network. In these networks, each of the peers hold a replica of the full ledger, execute operations on the ledger when achieving consensus, and share the user's transaction to other peers (Pappalaro et al, 2018). Regularly, each peer needs to make sure that the current local ledger is identical to the versions across the peer network (Gupta et al, 2019). Based on the growing interest and popularity of bitcoin, researchers started realizing the potential of such technology. Hence, many blockchain solutions are being developed in various fields (Gatteschi 2018), and therefore, the number of these solutions is rapidly grown. According to Gatteschi, et al, blockchain evolution can be categorized into three stages:

- Blockchain 1.0: refers to the usage of blockchain in cryptocurrency and simplifying transactions.

- Blockchain 2.0: introduces smart contracts, which are defined as programs that allow transactions under specific business rules; in other words, smart contracts enforce specific conditions on the transactions before being appended to the blockchain data structure. This addition revolutionizes decentralized solutions as smart contracts fulfill the needs that systems had for third parties.

- Blockchain 3.0: blockchain covers various areas and sectors; including governments, health, and education. Today. although the veracity and variety of literature on blockchain's application to educations has increased (Chen et al, 2018), it is still scattered and fragmented.

On the other hand, education is a fundamental factor of development. Besides improving people's quality of living, education also leads to broad social benefits to both individuals and societies (Ilhan, 2001). In fact, there is a strong evidence that the cognitive skills of the population are powerful indicators of individual earnings, distribution of income, and economic growth (Hanushek et al, 2007). Yet, education still suffers from several issues that limit the full utilization of the sector. Some instances of those issues are: redundant document exchange, scattered information, falsification of grades, and degree fraud.

Our solution consists on the development of a blockchain system whose participants are the students, schools, universities, affiliated companies, and the ministry of education. This centralization of information will provide new analytic features while granting a secure and immutable platform. Moreover, applying this technology to this field will enable a 
personalized and adequate follow of the learners. Applied on a large scale, this technology can offer standardized practices and policies which will considerably facilitate the communication and exchange of documents between the different participants. This paper presents, first, an overview of the blockchain technology, then introduce the current applications in education highlighting the capabilities of the blockchain technology, finally propose an application which focus on reducing the overhead as well as increasing transparent and security.

\section{Related Works}

The importance of education in the development and advancement of a country is undeniable (Johansson et al, 2010); hence, there have always been attempts to find innovative methods to apply various technologies in the field. Blockchain is no exception, in fact it was used in several higher education applications. Most of those solutions rely on a bitcoin blockchain approach (Fasso et al, 2020). NazarAl et al. suggested a platform for creating, verifying as well as sharing certificates. This method is led by Media Lab Learning Initiative at the Massachusetts Institute of Technology (MIT). Their methodology consists on solving issues related to the digitalization of academic certificates; however, the proposed solution does not investigate the potential capabilities of blockchain in a global higher education credits/grades management system.

Additionally, The National University of La Plata (UNLP) has started the development of a blockchain based framework that aims at verifying academic achievements; however, no academic paper releasing further details about the project has been published (Arganaraz et al, 2019). Argentinian College (CESYT) has adopted a similar approach in order to issue students' diplomas by utilizing Bitcoin-based Blockchain and cryptographic features; however, their approach lacks generalization and can hardly be implemented to follow students' complete academic careers as it does not offer the possibility to obtain credits for completed academic milestones.

Moreover, several institutions have shown interest in applying blockchain technology to manage student's academic affairs; for instance, partnering with the French Bitcoin startup Paymium, The Parisian Leonardo da Vinci Engineering School (ESILV) announced in 2016, a bitcoin-based certification mechanism (Das 2016). In 2015, using Blockchain technology, The Holverton School, a software engineering school in San Francisco, has allowed employers to verify applicants' academic credentials in order to reduce degree fraud (Coleman, 2015). However, both implementations have not yet been applied. 


\section{Methodology}

In this section, we will be using Hyperledger's schema to describe the blockchain network implementation. We will start by defining the model's structure, the chaincode, and the access control rules, then illustrate the system's architecture, and finally, state the advantages that our system offers over current systems.

\subsection{Hyperledger Project}

Hyperledger project has been released by the Linux Foundation in 2015. The project consists on an open source blockchain ecosystem that speeds up the development process of blockchain solutions by providing a distributed ledger (Dhillon et al, 2017). The power of Hyperledger project resides in its intent to provide a modular and extendable blockchain architecture that can be applied to various fields and diverse environments.

\subsection{Model}

\subsubsection{Participants}

The participants represent the different actors that take part of the educational business network (Cachin, 2016). This implementation makes use of the following 6 participants:

- Student: enroll in an institution, earn a grade in a class, and receive a diploma/certification

- Teacher/Professor: evaluate students' performance and access class statistics

- Parents/Tutors: access a student's view and request statistical information about a student's academic performance

- Institution: ranges from primary schools to universities, they enroll students, offer classes, and issue degrees

- Employers: Verify the authenticity of a degree and employee's academic information

- Administrator/Ministry of education: issues accounts, access educational statistics and request changes to alter the chaincode

Figure 1 shows a typical participant definition. In this case, we define a student-participant identified by his/her national identity number, a unique string identifier, which is reprented in the figure by "nin".. The student participant tracks the students' personal information as well as his/her academic career. 


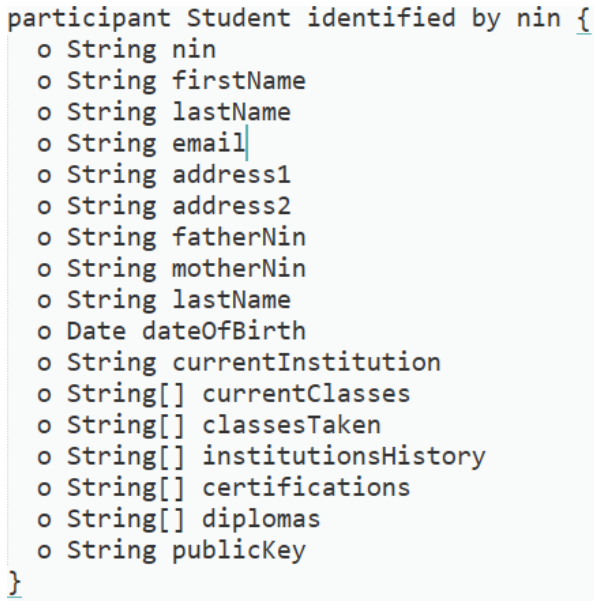

Figure 1. Typical Participant Definition.

\subsubsection{Assets}

Assets denote anything of value; this includes both physical and non-physical assets (Cachin, 2016). This implementation requires an asset per classes being taught and diploma/certificate obtained. These assets can be divided into two main categories:

- Classes: track detail of the courses, the students' enrollement, the faculty lectures, the students' grades, as well as a timestamp.

- Diplomas/Certificates: track documents' details, issuing institution, students' distinction, and a timestamp.

Figure 2 illustrates the definition of the course asset. In this implementation, we use a template to generate the class details, a timestamp in order to track temporal information about the class, the students' grades and their letter grades, reference the student passing the class, the instructor lectures the course and the institution offering it.

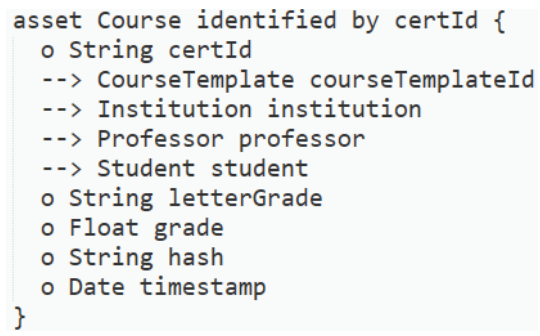

Figure 2. Typical Asset Definition. 


\subsubsection{Transacctions}

The transactions refer to the actions that can be executed by the participants in order to affect the assets held in the registry (Cachin, 2016). In this system, we define two operational transactions:

- Completing a Course: It can be whether passing or failing a class. In case of a successful completion, the asset is passed to the students containing their grades.

- Graduating: This transaction refers to the completion of academic milestones set by the administrator in the chaincode the attainement of a certificate or diploma.

Figure 3 shows the graduation transaction of a student. In this transaction, we transfer the ownership of a diploma-asset from an institution to the students completing the requirements.

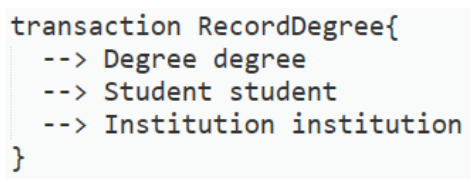

Figure 3. Typical Transaction Definition

\subsection{Chaincode}

The chaincode refers to a blockchain smart contract. It is a program that handles the business logic agreed upon by the network participants and takes care of initializing and managing the shared ledger through the transactions defined above (Cachin, 2016). This implementation defines the following functions:

- Student Creation and Update,

- Professor Creation and Update.

- Institution Creation and Update.

- Access to Statistics.

- Student/Professor Enrollment in institutions.

- Student/Professor Enrollment in courses.

- Student Completing a Course.

- Reception of Diploma.

- Evaluating a Student.

- Course Creation.

- Diploma Creation.

- Hiring a Professor.

- Verification of the Authentication of Degrees. 
The implementation consists of changing the owner of the certificate, setting the degree type, the institution affiliated to, initializing the timestamp to the current date and time, and finally update the asset from the asset registry using a promise. Figure 4 illustrates an overall structure and a design architecture of the described blockchain network. It shows the participants involved in this field, the assets that are in play, as well as the possible operations and actions that can be executed by the different actors.

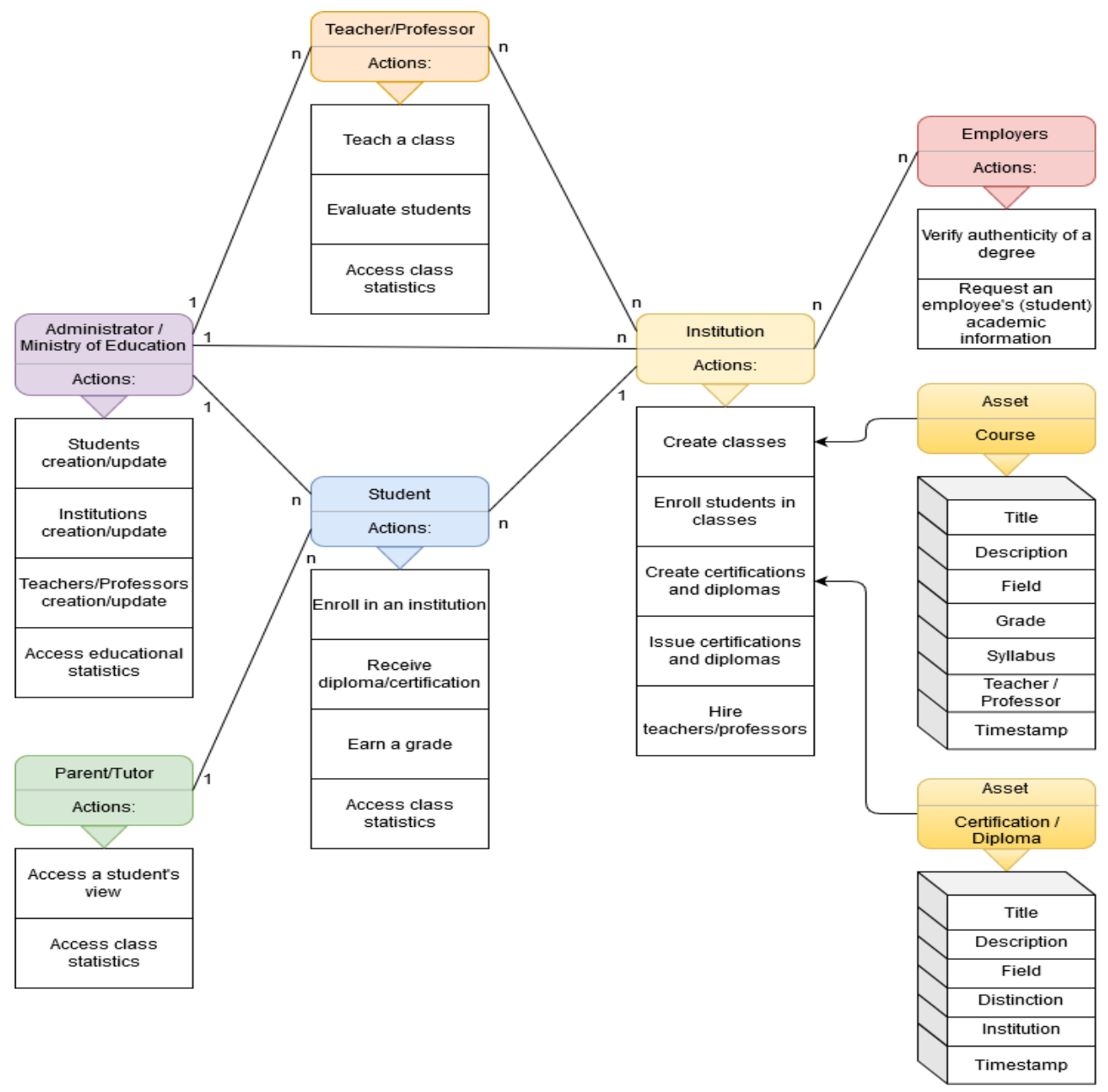

Figure 4. Overall Structure the Proposed Blockchain Network. 


\section{Future Work}

Although this implementation covers the main aspects of the educational field, there are few aspects that deserve to be handled in order to present a more complete image of the academic life. We cite the following as possible improvement to the system:

- Handle student clubs' positions and extracurricular activities.

- Allow inductrial employers to have access to class records in order to offer targeted hiring options.

- Include student's class work which can allow the generation of an entire academic portfolio.

- Incorporate machine learning capabilities to the statistical data collect in order to predict various information, such as: the time needed for a student to graduate, predict students' grades, and suggest statistically optimal career paths.

\section{Conclusion}

Blockchain is essentially constituted of a distributed ledger system and a world state database. This technology makes the use of cryptographic techniques and consensus validation algorithms to leverage decentralization, immutability, and complete traceability. When applied to education, those unique properties can offer several valuable improvements.

Blockchain technology as well as the described system offers numerous advantages over the currently adopted systems. Those advantages can be summarized as the following:

- Facilitate the application processes as institutions have access to the complete student academic records.

- Eradicate degree fraud as employers can trace employees' career paths.

- Unfalsifiable student grades thanks to immutability and cryptographic aspects of blockchain.

- Centralize data in one platform which offers numerous statistical capabilities.

Considering the current situation of COVID-19 outbreak in the world and the rise in the need of online and automated solutions in academia, this paper proposed an overview of a blockchain solution in education. The presented solution, which can be considered as a first step into applying blockchain into education, offers focused tracking of different student stages; starting from the student's first class to their job recruitment; thus, facilitating major procedures, providing useful statistical capabilities, and countering fraudulent activities. 


\section{References}

Argañaraz, Á. A., Mazzuchelli, A., Albanese, D., \& López, M. D. L. Á. (2019). Blockchain: un nuevo desafío para la contabilidad y auditoría.

Cachin, C. (2016, July). Architecture of the hyperledger blockchain fabric. In Workshop on distributed cryptocurrencies and consensus ledgers (Vol. 310, p. 4).

Chen, G.; Xu, B.; Lu, M.; Chen, N.-S. Exploring blockchain technology and its potential applications for education. Smart Learn. Environ. 2018, 5, 1.

Dhillon, V., Metcalf, D., \& Hooper, M. (2017). The hyperledger project. In Blockchain enabled applications (pp. 139-149). Apress, Berkeley, CA.

Fosso Wamba, S., Kala Kamdjoug, J. R., Epie Bawack, R., \& Keogh, J. G. (2020). Bitcoin, Blockchain and Fintech: a systematic review and case studies in the supply chain. Production Planning \& Control, 31(2-3), 115-142.

Gatteschi, V.; Lamberti, F.; Demartini, C.; Pranteda, C.; Santamaría, V. Blockchain and smart contracts for insurance: Is the technology mature enough? Future Internet, 2018, $10,20$.

Gupta, S., \& Sadoghi, M. (2019). Blockchain Transaction Processing.

Hanushek, E. A., \& Wößmann, L. (2007). The role of education quality for economic growth. The World Bank.

Herbert, J., \& Litchfield, A. (2015, January). A novel method for decentralised peer-to-peer software license validation using cryptocurrency blockchain technology. In Proceedings of the 38th Australasian computer science conference (ACSC 2015) (Vol. 27, p. 30).

Ilhan, O. (2001). The Role of Education in Economic Development: A Theoretical Perspective. SSRN Electronic Journal. 10.2139/ssrn.1137541.

Johansson, B., FOGELBERG-DAHM, M. A. R. I. E., \& Wadensten, B. (2010). Evidencebased practice: the importance of education and leadership. Journal of nursing management, 18(1), 70-77.

Coleman, L. Engineering School Simplifies Verifying Certificates Using the BlockchainCryptoCoinsNews, 2015, https:/www.cryptocoinsnews.com/engineering-schoolsimplifies-verifying-certificates-using-block-chain/

Li, Z., Barenji, A. V., \& Huang, G. Q. (2018). Toward a blockchain cloud manufacturing system as a peer to peer distributed network platform. Robotics and computer-integrated manufacturing, 54, 133-144.

Nakamoto, S. (2008). Bitcoin: A Peer-to-Peer Electronic Cash System.

Pappalardo, G., Di Matteo, T., Caldarelli, G., \& Aste, T. (2018). Blockchain inefficiency in the bitcoin peers network. EPJ Data Science, 7(1), 30.

Das, S. Parisian Engineering School Will Certify Diplomas on the BlockchainCryptoCoinsNews, 2016. https://www.cryptocoinsnews.com/parisian-engineeringschool-will-certify-diplomas-blockchain/. 